\title{
Superhalogens as Building Blocks of Ionic Liquids
}

\author{
Ambrish Kumar Srivastava ${ }^{*}$, Abhishek Kumar ${ }^{2}$, Neeraj Misra ${ }^{2}$ \\ ${ }^{1}$ Department of Physics, Deen Dayal Upadhyaya Gorakhpur University, Civil Lines, Gorakhpur \\ 273009, Uttar Pradesh, India \\ ${ }^{2}$ Department of Physics, University of Lucknow, University Road, Lucknow 226007, Uttar \\ Pradesh, India \\ *Corresponding author's e-mail: aks.ddugu@gmail.com; ambrish.phy@ddugu.ac.in
}

\section{SUPPLEMENTARY INFORMATION}

BMIM-PF $_{6}$

$\begin{array}{lrrr}\mathrm{N} & 2.416252000 & -0.491288000 & 0.481322000 \\ \mathrm{C} & 2.951827000 & -0.972916000 & -0.691703000 \\ \mathrm{C} & 2.215562000 & -2.053135000 & -1.049742000 \\ \mathrm{~N} & 1.243232000 & -2.214978000 & -0.090568000 \\ \mathrm{C} & 1.376013000 & -1.249804000 & 0.810476000 \\ \mathrm{C} & 2.800559000 & 0.750509000 & 1.168853000 \\ \mathrm{C} & 1.859619000 & 1.904604000 & 0.841083000 \\ \mathrm{C} & 1.827697000 & 2.279202000 & -0.638336000 \\ \mathrm{C} & 0.853984000 & 3.422169000 & -0.909251000 \\ \mathrm{C} & 0.123788000 & -3.151743000 & -0.162584000 \\ \mathrm{~F} & -3.382135000 & 0.172226000 & 0.739711000 \\ \mathrm{P} & -1.942880000 & 0.113319000 & 0.027691000 \\ \mathrm{~F} & -0.433844000 & 0.015393000 & -0.665196000 \\ \mathrm{~F} & -2.380512000 & -1.197270000 & -0.839308000 \\ \mathrm{~F} & -1.407448000 & 1.375470000 & 0.916169000 \\ \mathrm{~F} & -1.391682000 & -0.907556000 & 1.223988000 \\ \mathrm{~F} & -2.415317000 & 1.103053000 & -1.152095000 \\ \mathrm{H} & 3.799406000 & -0.506783000 & -1.163015000 \\ \mathrm{H} & 0.694943000 & -1.072553000 & 1.626752000 \\ \mathrm{H} & 2.813485000 & 0.539396000 & 2.239665000 \\ \mathrm{H} & 3.825374000 & 0.967985000 & 0.859797000 \\ \mathrm{H} & 2.294795000 & -2.711533000 & -1.897262000 \\ \mathrm{H} & -0.327781000 & -3.236081000 & 0.822407000 \\ \mathrm{H} & -0.624229000 & -2.758079000 & -0.850599000 \\ \mathrm{H} & 0.497490000 & -4.121017000 & -0.490300000 \\ \mathrm{H} & 0.846603000 & 1.657904000 & 1.171297000 \\ \mathrm{H} & 2.188595000 & 2.762183000 & 1.438898000\end{array}$




\begin{tabular}{lrrr}
$\mathrm{H}$ & 2.838221000 & 2.553472000 & -0.971070000 \\
$\mathrm{H}$ & 1.512553000 & 1.410816000 & -1.223760000 \\
$\mathrm{H}$ & -0.157526000 & 3.134014000 & -0.615324000 \\
$\mathrm{H}$ & 1.132289000 & 4.320733000 & -0.349464000 \\
$\mathrm{H}$ & 0.836740000 & 3.678447000 & -1.970854000 \\
\hline
\end{tabular}

BMIM-BF $_{4}$

\begin{tabular}{rrrr}
\hline $\mathrm{N}$ & 0.602437000 & -1.540970000 & 0.702408000 \\
$\mathrm{C}$ & 0.762809000 & -2.534278000 & -0.237526000 \\
$\mathrm{C}$ & -0.447807000 & -2.739271000 & -0.809773000 \\
$\mathrm{~N}$ & -1.327651000 & -1.863858000 & -0.213729000 \\
$\mathrm{C}$ & -0.667387000 & -1.146772000 & 0.688717000 \\
$\mathrm{C}$ & 1.682316000 & -0.906358000 & 1.473955000 \\
$\mathrm{C}$ & 2.230540000 & 0.347796000 & 0.801025000 \\
$\mathrm{C}$ & 2.728317000 & 0.133774000 & -0.624710000 \\
$\mathrm{C}$ & 3.290174000 & 1.420088000 & -1.222417000 \\
$\mathrm{C}$ & -2.738363000 & -1.696934000 & -0.553926000 \\
$\mathrm{~F}$ & -2.539120000 & 1.284364000 & 0.086550000 \\
$\mathrm{~F}$ & -1.270746000 & 3.176642000 & -0.343073000 \\
$\mathrm{~F}$ & -0.656226000 & 1.694469000 & 1.327543000 \\
$\mathrm{~F}$ & -0.463186000 & 1.078078000 & -0.879315000 \\
$\mathrm{H}$ & 1.718359000 & -2.994098000 & -0.420086000 \\
$\mathrm{H}$ & -1.070102000 & -0.334464000 & 1.276426000 \\
$\mathrm{H}$ & 1.279290000 & -0.670568000 & 2.459552000 \\
$\mathrm{H}$ & 2.454622000 & -1.669552000 & 1.597239000 \\
$\mathrm{H}$ & -0.756909000 & -3.418922000 & -1.584778000 \\
$\mathrm{H}$ & -3.045813000 & -0.699681000 & -0.241795000 \\
$\mathrm{H}$ & -2.842402000 & -1.772548000 & -1.635217000 \\
$\mathrm{H}$ & -3.332110000 & -2.469380000 & -0.064042000 \\
$\mathrm{H}$ & 1.454627000 & 1.115254000 & 0.809976000 \\
$\mathrm{H}$ & 3.049500000 & 0.707548000 & 1.434628000 \\
$\mathrm{H}$ & 3.493873000 & -0.654571000 & -0.642305000 \\
$\mathrm{H}$ & 1.895667000 & -0.204266000 & -1.249128000 \\
$\mathrm{H}$ & 2.518063000 & 2.192951000 & -1.246888000 \\
$\mathrm{H}$ & 4.131698000 & 1.797135000 & -0.633026000 \\
$\mathrm{H}$ & 3.640268000 & 1.260864000 & -2.245020000 \\
$\mathrm{~B}$ & -1.238446000 & 1.855568000 & 0.029260000 \\
\hline & & & \\
\hline
\end{tabular}

\section{BMIM-BeF ${ }_{3}$}

$\begin{array}{lrrr}\mathrm{N} & -1.140451000 & -1.236527000 & 0.512349000 \\ \mathrm{C} & -2.024761000 & -1.656875000 & -0.456532000 \\ \mathrm{C} & -2.728012000 & -0.566616000 & -0.849191000 \\ \mathrm{~N} & -2.263256000 & 0.497141000 & -0.110700000 \\ \mathrm{C} & -1.294335000 & 0.072797000 & 0.692893000\end{array}$




\begin{tabular}{lrrr}
$\mathrm{C}$ & -0.099514000 & -2.052021000 & 1.156449000 \\
$\mathrm{C}$ & 1.306056000 & -1.602644000 & 0.769470000 \\
$\mathrm{C}$ & 1.557331000 & -1.598809000 & -0.737266000 \\
$\mathrm{C}$ & 2.997514000 & -1.223458000 & -1.077396000 \\
$\mathrm{C}$ & -2.581522000 & 1.904472000 & -0.328366000 \\
$\mathrm{H}$ & -2.077495000 & -2.683452000 & -0.774951000 \\
$\mathrm{H}$ & -0.660535000 & 0.709674000 & 1.313013000 \\
$\mathrm{H}$ & -0.251245000 & -1.996857000 & 2.235905000 \\
$\mathrm{H}$ & -0.283734000 & -3.081344000 & 0.841917000 \\
$\mathrm{H}$ & -3.506726000 & -0.457309000 & -1.583928000 \\
$\mathrm{H}$ & -2.453836000 & 2.440489000 & 0.610156000 \\
$\mathrm{H}$ & -1.883193000 & 2.300778000 & -1.064822000 \\
$\mathrm{H}$ & -3.615459000 & 1.986639000 & -0.660834000 \\
$\mathrm{H}$ & 1.496151000 & -0.610042000 & 1.190719000 \\
$\mathrm{H}$ & 2.004560000 & -2.285040000 & 1.266452000 \\
$\mathrm{H}$ & 1.319124000 & -2.589941000 & -1.146962000 \\
$\mathrm{H}$ & 0.884454000 & -0.879610000 & -1.216302000 \\
$\mathrm{H}$ & 3.227033000 & -0.213067000 & -0.728678000 \\
$\mathrm{H}$ & 3.705933000 & -1.920895000 & -0.618591000 \\
$\mathrm{H}$ & 3.157285000 & -1.244631000 & -2.157869000 \\
$\mathrm{Be}$ & 1.336538000 & 1.865220000 & 0.130563000 \\
$\mathrm{~F}$ & 2.747644000 & 2.064896000 & -0.152492000 \\
$\mathrm{~F}$ & 0.301609000 & 1.606663000 & -0.923151000 \\
$\mathrm{~F}$ & 0.797517000 & 1.726035000 & 1.535711000 \\
\hline & & &
\end{tabular}

BMIM-LiF $_{2}$

\begin{tabular}{rrrr}
\hline $\mathrm{N}$ & 0.174693000 & -0.422556000 & 0.745455000 \\
$\mathrm{C}$ & 0.300570000 & -1.780434000 & 0.938108000 \\
$\mathrm{C}$ & -0.798006000 & -2.364019000 & 0.396103000 \\
$\mathrm{~N}$ & -1.574669000 & -1.350708000 & -0.120993000 \\
$\mathrm{C}$ & -0.966584000 & -0.187942000 & 0.102674000 \\
$\mathrm{C}$ & 1.132962000 & 0.625287000 & 1.136012000 \\
$\mathrm{C}$ & 2.173431000 & 0.882088000 & 0.053235000 \\
$\mathrm{C}$ & 3.063570000 & -0.308521000 & -0.295277000 \\
$\mathrm{C}$ & 4.135515000 & 0.046700000 & -1.321990000 \\
$\mathrm{C}$ & -2.869613000 & -1.482660000 & -0.790207000 \\
$\mathrm{H}$ & 1.150653000 & -2.206581000 & 1.442241000 \\
$\mathrm{H}$ & -1.368594000 & 0.780553000 & -0.176600000 \\
$\mathrm{H}$ & 0.535246000 & 1.531826000 & 1.295133000 \\
$\mathrm{H}$ & 1.596333000 & 0.294216000 & 2.068921000 \\
$\mathrm{H}$ & -1.093846000 & -3.397465000 & 0.339791000 \\
$\mathrm{H}$ & -3.234693000 & -0.464520000 & -0.983599000 \\
$\mathrm{H}$ & -2.737691000 & -2.035404000 & -1.721140000 \\
$\mathrm{H}$ & -3.555058000 & -2.018367000 & -0.132787000 \\
$\mathrm{H}$ & 1.657051000 & 1.248366000 & -0.840598000 \\
$\mathrm{H}$ & 2.791473000 & 1.714719000 & 0.405849000
\end{tabular}




\begin{tabular}{lrrr}
$\mathrm{H}$ & 3.541096000 & -0.685496000 & 0.618500000 \\
$\mathrm{H}$ & 2.450467000 & -1.128511000 & -0.686854000 \\
$\mathrm{H}$ & 3.682020000 & 0.406017000 & -2.250351000 \\
$\mathrm{H}$ & 4.791196000 & 0.837651000 & -0.946363000 \\
$\mathrm{H}$ & 4.757948000 & -0.817754000 & -1.565546000 \\
$\mathrm{Li}$ & -2.323213000 & 2.771584000 & -0.135604000 \\
$\mathrm{~F}$ & -3.113414000 & 1.439621000 & -0.875915000 \\
$\mathrm{~F}$ & -0.800498000 & 2.722140000 & 0.637808000 \\
\hline
\end{tabular}

BMIM-BO $_{2}$

\begin{tabular}{rrrr}
\hline $\mathrm{N}$ & -0.357136000 & -0.503321000 & 0.824847000 \\
$\mathrm{C}$ & -0.922209000 & -1.708385000 & 1.176570000 \\
$\mathrm{C}$ & -2.097048000 & -1.810665000 & 0.507158000 \\
$\mathrm{~N}$ & -2.220361000 & -0.662269000 & -0.246457000 \\
$\mathrm{C}$ & -1.156160000 & 0.115273000 & -0.042929000 \\
$\mathrm{C}$ & 0.954388000 & 0.013486000 & 1.262440000 \\
$\mathrm{C}$ & 1.999185000 & -0.101677000 & 0.161142000 \\
$\mathrm{C}$ & 2.277906000 & -1.530629000 & -0.296456000 \\
$\mathrm{C}$ & 3.358279000 & -1.595508000 & -1.372277000 \\
$\mathrm{C}$ & -3.331648000 & -0.310502000 & -1.120877000 \\
$\mathrm{H}$ & -0.444117000 & -2.382144000 & 1.866608000 \\
$\mathrm{H}$ & -0.964683000 & 1.121536000 & -0.509007000 \\
$\mathrm{H}$ & 0.833093000 & 1.063495000 & 1.543395000 \\
$\mathrm{H}$ & 1.233080000 & -0.567970000 & 2.144076000 \\
$\mathrm{H}$ & -2.844323000 & -2.585521000 & 0.504302000 \\
$\mathrm{H}$ & -3.122342000 & 0.663878000 & -1.559615000 \\
$\mathrm{H}$ & -3.429629000 & -1.054457000 & -1.911702000 \\
$\mathrm{H}$ & -4.254188000 & -0.257919000 & -0.542445000 \\
$\mathrm{H}$ & 1.684853000 & 0.519673000 & -0.684594000 \\
$\mathrm{H}$ & 2.912612000 & 0.362309000 & 0.545393000 \\
$\mathrm{H}$ & 2.580833000 & -2.137461000 & 0.566594000 \\
$\mathrm{H}$ & 1.356383000 & -1.982493000 & -0.683960000 \\
$\mathrm{H}$ & 3.066704000 & -1.015476000 & -2.252402000 \\
$\mathrm{H}$ & 4.302243000 & -1.184298000 & -1.003633000 \\
$\mathrm{H}$ & 3.541822000 & -2.624115000 & -1.692033000 \\
$\mathrm{O}$ & -0.555125000 & 2.638080000 & -1.020853000 \\
$\mathrm{O}$ & 1.034642000 & 3.211507000 & 0.836344000 \\
$\mathrm{~B}$ & 0.251568000 & 2.999008000 & -0.110452000 \\
\hline & & & \\
\hline
\end{tabular}

BMIM-NO $_{3}$

\begin{tabular}{rrrr}
\hline $\mathrm{N}$ & -0.993868000 & 1.314215000 & -0.599503000 \\
$\mathrm{C}$ & -1.841760000 & 1.873381000 & 0.332919000 \\
$\mathrm{C}$ & -2.618182000 & 0.871471000 & 0.813476000 \\
$\mathrm{~N}$ & -2.228050000 & -0.280757000 & 0.168801000 \\
$\mathrm{C}$ & -1.240199000 & 0.009205000 & -0.668661000 \\
$\mathrm{C}$ & 0.124499000 & 1.981492000 & -1.281531000
\end{tabular}




\begin{tabular}{lrrr}
$\mathrm{C}$ & 1.476282000 & 1.454437000 & -0.809169000 \\
$\mathrm{C}$ & 1.706063000 & 1.608507000 & 0.692323000 \\
$\mathrm{C}$ & 3.064979000 & 1.062236000 & 1.119455000 \\
$\mathrm{C}$ & -2.666481000 & -1.641743000 & 0.458909000 \\
$\mathrm{H}$ & -1.814590000 & 2.922305000 & 0.571835000 \\
$\mathrm{H}$ & -0.688680000 & -0.743095000 & -1.235955000 \\
$\mathrm{H}$ & -0.005538000 & 1.840303000 & -2.356104000 \\
$\mathrm{H}$ & 0.021284000 & 3.047265000 & -1.067052000 \\
$\mathrm{H}$ & -3.399755000 & 0.878401000 & 1.553371000 \\
$\mathrm{H}$ & -2.026160000 & -2.322233000 & -0.100352000 \\
$\mathrm{H}$ & -2.527387000 & -1.835889000 & 1.521328000 \\
$\mathrm{H}$ & -3.712389000 & -1.762871000 & 0.175335000 \\
$\mathrm{H}$ & 1.584075000 & 0.403039000 & -1.095527000 \\
$\mathrm{H}$ & 2.244724000 & 2.001099000 & -1.367137000 \\
$\mathrm{H}$ & 1.616125000 & 2.668787000 & 0.965985000 \\
$\mathrm{H}$ & 0.929563000 & 1.059765000 & 1.234170000 \\
$\mathrm{H}$ & 3.124974000 & -0.007376000 & 0.902661000 \\
$\mathrm{H}$ & 3.880027000 & 1.572268000 & 0.595540000 \\
$\mathrm{H}$ & 3.217808000 & 1.198266000 & 2.192640000 \\
$\mathrm{~N}$ & 1.050125000 & -1.991715000 & -0.102960000 \\
$\mathrm{O}$ & 0.448656000 & -1.497113000 & 0.881597000 \\
$\mathrm{O}$ & 0.375641000 & -2.291010000 & -1.131533000 \\
$\mathrm{O}$ & 2.266611000 & -2.152646000 & -0.087744000 \\
\hline & & &
\end{tabular}

\section{BMIM-CI}

$\begin{array}{rrrr}\mathrm{N} & -0.761786000 & 1.269313000 & -0.322969000 \\ \mathrm{C} & -1.951011000 & 1.816907000 & 0.112156000 \\ \mathrm{C} & -2.767397000 & 0.784239000 & 0.436706000 \\ \mathrm{C} & -2.059554000 & -0.372931000 & 0.193520000 \\ \mathrm{C} & -0.851791000 & -0.058206000 & -0.268319000 \\ \mathrm{C} & 0.413405000 & 2.017649000 & -0.799572000 \\ \mathrm{C} & 1.713672000 & 1.238247000 & -0.645121000 \\ \mathrm{C} & 2.106495000 & 0.941889000 & 0.801933000 \\ \mathrm{C} & 3.405437000 & 0.146032000 & 0.879209000 \\ \mathrm{C} & -2.503774000 & -1.748358000 & 0.415665000 \\ \mathrm{H} & -2.109924000 & 2.880748000 & 0.156450000 \\ \mathrm{H} & -0.074025000 & -0.829334000 & -0.485837000 \\ \mathrm{H} & 0.237191000 & 2.277186000 & -1.846306000 \\ \mathrm{H} & 0.447136000 & 2.942675000 & -0.218261000 \\ \mathrm{H} & -3.773097000 & 0.774449000 & 0.820630000 \\ \mathrm{H} & -1.653774000 & -2.406724000 & 0.216903000 \\ \mathrm{H} & -2.824514000 & -1.859534000 & 1.451323000 \\ \mathrm{H} & -3.326947000 & -1.979917000 & -0.260483000 \\ \mathrm{H} & 1.667760000 & 0.300319000 & -1.209454000 \\ \mathrm{H} & 2.493684000 & 1.843353000 & -1.119524000 \\ \mathrm{H} & 2.198528000 & 1.886571000 & 1.353511000\end{array}$




$\begin{array}{lrrr}\mathrm{H} & 1.318648000 & 0.359889000 & 1.291215000 \\ \mathrm{H} & 3.272641000 & -0.826346000 & 0.397649000 \\ \mathrm{H} & 4.225395000 & 0.677987000 & 0.385540000 \\ \mathrm{H} & 3.694917000 & -0.028485000 & 1.918063000 \\ \mathrm{Cl} & 0.974444000 & -2.536347000 & -0.443602000\end{array}$

